\title{
Case Report: Surgical Treatment of Drug-Resistant Temporal Lobe Epilepsy
}

\section{Fernando Cendes*, M.D}

* To whom correspondence should be addressed: Department of Neurology and Neurosurgery; Montreal Neurological Institute and Hospital; McGill University, 3801 University Street, Montreal, QC, Canada H3A 2B4

\section{INTRODUCTION}

In recent years, there has been increasing interest in the surgical treatment of temporal lobe epilepsy (TLE). In some patients inadequately controlled by anticonvulsant medication $(1,2)$, surgery offers a safe and effective treatment modality capable of greatly reducing the frequency of epileptic seizures.

In the first group of patients undergoing this form of treatment, gross structural lesions were identified on regular skull x-rays and pneumoencephalograms. However, these methods were relatively insensitive and therefore uninformative in a large number of patients. Bailey and Gibbs (3) in Chicago, and Penfield and Jasper (4) at the Montreal Neurological Institute were the first to perform surgical resection for the treatment of complex partial seizures using a unilateral temporal interictal electroencephalogram (EEG) spike focus as the major indication for anterior temporal lobe resection. Today, anterior temporal lobe removal has become the most common, and successful, surgical treatment available for epilepsy $(1,2,5)$.

The primary objective of presurgical evaluation is to identify the region responsible for generating the patient's habitual seizures, and to determine whether the intended resection can be performed without producing unacceptable neurological deficits.

The following report is about a common neurological condition, recognized over a century ago but still often misdiagnosed and therefore not properly treated. The patient described in this case report clearly illustrates the syndrome of mesial temporal lobe epilepsy (6).

\section{CASE REPORT}

\section{Patient Profile and Reason for Visit}

A 22 year-old woman was admitted to the Montreal Neurological Hospital for investigation of longstanding epileptic seizures uncontrolled by appropriate anticonvulsant medication.

\section{Clinical Presentation}

When the patient was eight months old, she experienced an acute episode of febrile convulsions with no 
apparent complications. She did not receive medication at the time, but was symptom-free until she was nine years old, when her mother noticed brief "absence-like" episodes, manifested by staring and unresponsiveness for a few seconds. Since these episodes were at first infrequent, and the neurological exam and EEG were normal, the attending physician decided against medication. However, the brief spells progressively became more frequent, even though another EEG showed no epileptic abnormalities.

One year later, she was administered carbamazepine and was free of seizures for a few months. However, she subsequently started to experience typical complex partial seizures. Several anticonvulsant regimens were attempted after carbamazepine monotherapy without achieving seizure control, first with phenytoin alone, then with a combination of the two drugs. A few years later, valproate, then clobazam were administered with carbamazepine and phenytoin, but this simply increased the patient's side effects without controlling her attacks. She often presented with symptoms of drug toxicity and had to be hospitalized twice because of acute ataxia, nystagmus, and mental confusion.

Seventy-five percent of these seizures were preceded by an aura (simple partial seizure) which the patient described as a feeling of unreality, accompanied by palpitations and an epigastric sensation similar to "a tickle rising toward her chest." These aurae typically lasted 10-15 seconds and would either resolve or progress into a full complex partial seizure. During these episodes, witnesses say the patient stared, had orofacial automatisms, and displayed purposeless finger and hand movements. Occasionally, she would walk around aimlessly for a period lasting anywhere from a few seconds to a minute. Postictally, she was tired and slow to respond, but showed no specific neurological deficits. Since the onset of the patient's complex partial seizures, only two episodes of generalized tonic-clonic seizures have been reported.

The patient's mother's pregnancy was uneventful; other than febrile convulsions at eight months of age, the remainder of the patient's past medical history is unremarkable. She has completed high school and attended college for 18 months, after which she had to quit because of her attacks.

The patient's general and neurological examination were within normal limits.

\section{INVESTIGATION}

\section{Neuropsychological Evaluation}

The neuropsychological evaluation revealed a low-average intelligence (full-scale I.Q. of 80, Wechsler Adult Intelligence Scale) and low scores for tests assessing verbal memory. These results seem to indicate that the patient has an impairment of the dominant temporal lobe, presumably the left lobe, based on her righthandedness and the results of the dichotic-listening test $(7,8)$.

\section{EEG}

Initial EEGs revealed intermittent slow wave abnormalities over the left temporal region, which, at times, presented as intermittent rhythmic delta activity (Fig. 1). On scalp EEG recordings, the occurrence of continuous or intermittent focal slow waves such as these is an indication that there might be localized structural cerebral pathology.

Further recordings showed sharp slow waves and spike waves over the left temporal lobe, with maximal intensity over the mid-inferomesial temporal region (Fig. 2). These "spiky" waveforms, referred to as "epileptic" or "epileptiform" discharges, are due to abnormal, synchronous cerebral neuronal activity and are seen in the interictal EEGs of most people with epilepsy. The presence of interictal spikes is currently considered an important criterion in the diagnosis of epilepsy, and the anatomic location from which they originate is helpful for diagnosing both the type of the seizure and the location of its origin (1-3). 
She then underwent intensive video-EEG monitoring of her seizures after tapering off the anticonvulsant medication. A firm diagnosis of epilepsy may not be obtained by examining the patient at one moment in time or recording the electrical activity of the brain for only a few minutes. It is therefore more effective to employ prolonged video-EEG monitoring, which has become the most prevalent specialized recording technique used at epilepsy surgery centers $(1,2)$. Ictal recordings are important for defining the area of the cortex where seizures originate, and for documenting the behavioral manifestations that accompany the electrographic changes $(1,2)$. Three of the patient's habitual seizures were recorded, with the initial ictal EEG changes occurring over the left mid-inferomesial temporal region (Fig. 3).

\section{Neuroimaging}

A computed tomography (CT) of the patient's brain performed a few years before her hospital admission was normal. The magnetic resonance imaging (MRI) scans demonstrated atrophy of the left hippocampus by qualitative and quantitative analyses. In addition, there was an abnormal signal intensity over the left hippocampus on T2-weighted and inversion-recovery T1-weighted images (Fig. 4). These findings correlate highly with the diagnosis of hippocampal sclerosis on histopathology, as demonstrated by several studies (919).

\section{MANAGEMENT AND OUTCOME}

Surgical excision of the epileptogenic brain tissue was proposed since the patient's long-standing history of complex partial seizures was not controlled by medication; her history and clinical symptomatology were compatible with temporal lobe epilepsy; and the EEG, MRI, and neuropsychological findings all localized the origin of the seizures to the left mesial temporal lobe.

After a left temporal-parietal craniotomy was performed (Fig. 5), three rows of electrodes were positioned along the cortical surface of the first, second, and third temporal gyri, with one set over the supra-Sylvian region in order to map the interictal epileptiform abnormalities. In addition, two electrodes were inserted through the second temporal gyrus toward the amygdala and anterior hippocampal region. An intraoperative EEG (ECoG) was then recorded in order to map the interictal epileptiform abnormality. No epileptic disturbance was recorded from the cortical surface. Conversely, frequent, and at times almost continuous, sharp waves were recorded from the anterior hippocampal region.

An excision of $4.5 \mathrm{~cm}$ along the first temporal gyrus and $5.5 \mathrm{~cm}$ along the third temporal gyrus was carried out, which included the amygdala and approximately $2 \mathrm{~cm}$ of the hippocampal formation. The anterior hippocampus was found to be hard and whitish. A post-excision ECoG detected residual spikes from the posterior cortical margin of the resection.

Postoperative histopathological examination of resected specimens revealed gliosis and profound neuronal loss of the hippocampal pyramidal neurons maximally over the CA1 and CA3 fields $(6,20,21)$. Sections containing temporal cortex, subiculum, and white matter revealed no abnormality.

This patient has been seizure-free since the surgery two years ago. She is back to work and now plans to return to school to obtain a college degree.

\section{DISCUSSION}

The presurgical localization of intractable epilepsy depends upon the analysis of clinical seizure patterns in association with extensive EEG investigation. EEG recording of seizures is generally considered necessary for seizure localization before surgical treatment in patients who do not have an obvious "foreign tissue" lesion. Radiological and neuropsychological studies are also very important. When the findings of different modalities are not completely congruous or when there are contradictory results from surface interictal and 
ictal recordings, monitoring with surgically implanted depth electrodes may be required $(22,23)$.

In recent years, there has been increasing recognition of the role of structural lesions secondary to injury to the central nervous system. By far, the most common example of this is mesial temporal atrophy associated with neuronal loss and gliosis, described as mesial temporal sclerosis (MTS) $(6,20,21,24,25)$. This entity, strongly associated with febrile seizures and other insults during early development (usually under age four), often produces a mesial temporal ictal onset, and is amenable to treatment by selective mesial temporal resection. Other pathologic substrates associated with intractable temporal lobe seizures include small lesions, such as neuronal migration disorders, vascular malformations, hamartomas, and low-grade gliomas. In patients who underwent surgical resection for epilepsy in the past, the lesions were most often occult, being discovered only at operation or in the surgical specimen. However, modern neuroimaging techniques, particularly MRI, have revolutionized the detection of such lesions.

The demonstration by MRI of atrophy and signal changes suggesting MTS has streamlined the presurgical evaluation of patients with TLE (9-19). In addition, MRI readily identifies other pathologies causing TLE, even though fine contiguous slices are required to detect the often small lesions.

The presence of unequivocal asymmetries or small lesions, found on imaging, greatly facilitate preoperative lateralization of TLE. The congruence of such imaging abnormalities with EEG and neuropsychological findings improves the localization accuracy so that in many individuals, invasive recordings with intracranial electrodes are unnecessary, and ultimately improves the prognosis of patients with drug-resistant TLE through successful surgical treatment.

\section{REFERENCES}

1. Engel J, Jr. Surgical treatment of the epilepsies. New York, NY: Raven Press; 1987.

2. Luders H. Epilepsy Surgery. New York, NY: Raven Press; 1991.

3. Bailey P, Gibbs FA. Surgical treatment of psychomotor epilepsy. JAMA 145: 365-370; 1951.

4. Penfield W, Jasper H. Epilepsy and the Functional Anatomy of the Human Brain. Boston, MA: Little, Brown; 1954.

5. Wieser HG, Elger CE. Presurgical Evaluation of Epileptics: Basics, Techniques, Implications. Berlin: Springer-Verlag; 1987.

6. Gloor P. Mesial temporal sclerosis: historical background and an overview from a modern perspective. In: Luders H, ed. Epilepsy surgery. New York, NY: Raven Press, 689-703; 1991.

7. Jones-Gotman M. Localization of lesions by neuropsychological testing. Epilepsia 32 Suppl 5: S41-S52; 1991.

8. Jones-Gotman M. Presurgical neuropsychological evaluation for localization and lateralization of seizure focus. In: Luders H, ed. Epilepsy Surgery. New York, NY: Raven Press, 469-475; 1991.

9. Berkovic SF, Andermann F, Olivier A, et al. Hippocampal sclerosis in temporal lobe epilepsy demonstred by magnetic resonance imaging. Annals of Neurology 29: 175-182; 1991.

10. Cascino GD, Jack CR, Parisi JE, et al. Magnetic resonance imaging-based volume studies in temporal lobe epilepsy: pathological correlations. Annals of Neurology 30: 31-36; 1991. 
11. Cendes F, Andermann F, Dubeau F, et al. Early childhood prolonged febrile convulsions, atrophy and sclerosis of mesial structures and temporal lobe epilepsy: an MRI volumetric study. Neurology 43: 10831087; 1993.

12. Cendes F, Andermann F, Gloor P, et al. MRI volumetric measurements of amygdala and hippocampus in temporal lobe epilepsy. Neurology 43: 719-725; 1993.

13. Cendes F, Andermann F, Gloor P, Olivier A, Evans A, Peters T. The role of MRI volumetric studies in the investigation of patients with intractable epilepsy. In: Shorvon SD, Fish DR, Andermann F, Bydder GM, Stefan H, eds. Magnetic Resonance Scaning and Epilepsy. New York, NY: Plenum Press, 57-61; 1994.

14. Cendes F, Leproux F, Melanson D, et al. MRI of amygdala and hippocampus in temporal lobe epilepsy. Journal of Computer Assisted Tomography 17: 206-210; 1993.

15. Jack CR, Sharbrough FW, Cascino GD, et al. Magnetic resonance image-based hippocampal volumetry: correlation with outcome after temporal lobectomy. Annals of Neurology 31: 138-146; 1992.

16. Jack CR, Sharbrough FW, Twomey CK, et al. Temporal lobe seizures: lateralization with MR volume measurements of the hippocampal formation. Radiology 175: 423-429; 1990.

17. Kuzniecky R, de la Sayette V, Ethier R, et al. Magnetic resonance imaging in temporal lobe epilepsy: pathological correlations. Annals of Neurology 22: 341-347; 1987.

18. Lencz T, McCarthy G, Bronen RA, et al. Quantitative magnetic resonance imaging in temporal lobe epilepsy: relationship to neuropathology and neuropsychological function. Annals of Neurology 31: 629-637; 1992.

19. Sperling MR, Wilson G, Engel J, Jr., Babb TL, Phelps M, Bradley W. Magnetic resonance imaging in intractable partial epilepsy: correlative studies. Annals of Neurology 20: 57-62; 1986.

20. Babb TL, Brown WJ. Pathological findings in epilepsy. In: Engel J, Jr. ed. Surgical treatment of the epilepsies. New York, NY: Raven Press, 511-540; 1987.

21. Bruton CJ. The neuropathology of temporal lobe Epilepsy. New York, NY: Oxford University Pressl; 1988.

22. Gloor P. Contributions of electroencephalography and electrocorticography to the neurosurgical treatment of the epilepsies. In: Purpura DP, Penry JK, Walter RD, eds. Neurosurgical manangement of the epilepsies. Advances of Neurology, Vol 8. New York, NY: Raven Press, 59-105; 1975.

23. So N. Depth electrodes studies in mesial temporal epilepsy. In: Luders H, ed. Epilepsy Surgery. New York, NY: Raven Press, 371-384; 1991.

24. Meencke HJ, Veith G. Hippocampal sclerosis in epilepsy. In: Luders H, ed. Epilepsy Surgery. New York, NY: Raven Press, 689-703; 1991.

25. Spielmeyer W. Die Pathogenese des epileptischen Krampfes. Ztschr Neuro Psychiat 109: 501-519; 1927.

\section{BIOGRAPHY}


Fernando Cendes received his M.D. at the Federal University of Goias (Goiania, Brazil) in 1986 and completed his residency in Neurology at the State University of Campinas in 1989. He completed an EEG fellowship in 1991 and an epilepsy fellowship in 1994, both at the M.N.I. He is currently in his third year of a $\mathrm{Ph} . \mathrm{D}$. program in the department of Neurology and Neurosurgery and is a research fellow in the Epilepsy Service at the M.N.I. His research interests are in the fields of Neurophysiology and Neuroimaging in the context of the presurgical evaluation of epilepsies.

Copyright (C) 1995 by MJM 